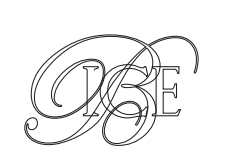

\title{
LA VISIÓN EMPRESARIAL DE LA RELACIÓN COMERCIAL Y DE INVERSIÓN ENTRE LA UE Y CANADÁ
}

Las buenas y añejas relaciones comerciales entre la UE y Canadá, cristalizadas en el acuerdo CETA, permiten a las empresas europeas nuevas oportunidades de negocio. Para ello, cuentan con el apoyo de la Cámara de Comercio de la Unión Europea en Canadá, que les ofrece unos recursos clave a través de tres pilares: defensa, información y representación de sus miembros.

En este artículo se revisarán las oportunidades que ofrece Canadá en materia de contratación pública y comercio electrónico, así como en otros sectores industriales de interés relacionados con la transición ecológica, la industria de alimentación y bebidas, y las tecnologías de digitalización.

Palabras clave: CETA, EUCCAN, contratos públicos, comercio electrónico, alimentación, tecnología. Clasificación JEL: F13, H57, L66, Q55.

\section{Introducción}

La EUCCAN (Cámara de Comercio de la Unión Europea en Canadá) aglutina empresas privadas de ambos lados del Atlántico y actúa como portavoz de los intereses empresariales de la UE en Canadá.

La UE y Canadá mantienen relaciones bastante añejas. Los líderes de nuestras naciones han reiterado esta relación en la última cumbre UE-Canadá celebrada en Bruselas. Nuestra sociedad está unida por muchos intereses comunes, valores democráticos y fuertes lazos entre las personas.

\footnotetext{
* Directora Ejecutiva de EUCCAN (Cámara de Comercio de la Unión Europea en Canadá).

Versión de octubre de 2021.

DOI: https://doi.org/10.32796/bice.2021.3140.7300
}

EI CETA (acuerdo comercial entre la UE y Canadá), en vigor desde hace cuatro años, es una extensión empresarial y comercial de esta buena relación. Los resultados positivos demuestran que este acuerdo comercial global es de gran ayuda para las empresas a la hora de aprovechar nuevas oportunidades de negocio en términos de comercio e inversión a ambos lados del Atlántico.

La pandemia de la COVID-19 y el cambio de la economía mundial han demostrado una vez más que la UE y Canadá tienen la misma visión. Ambas se han comprometido a trabajar en una recuperación socioeconómica sostenible e integradora tras la pandemia de la COVID-19 y a abordar los retos del cambio climático y el medioambiente.

La sostenibilidad ya no es una opción, a medida que aumenta la presión sobre los $\triangleright$ 
recursos, las mentalidades y los hábitos deben cambiar. Para evitar las graves consecuencias del calentamiento global, necesitamos limitar el calentamiento a 2 o incluso 1,5 grados. El Acuerdo de París para hacer frente al cambio climático, aceptado por 195 países, establece una nueva dirección y escala para la acción climática mundial. Pero como los marcos son solo el punto de partida, los Gobiernos, las empresas y las comunidades tienen que tomar medidas para pasar a una economía de carbono cero. Nosotros, en la EUCCAN, estamos dispuestos a contribuir a esta recuperación global hacia economías verdes, innovadoras, inclusivas y resilientes.

En las siguientes secciones, y tras presentar a la EUCCAN, se procederá a realizar un análisis de las oportunidades que se abren para las empresas europeas en materia de contratación pública y comercio electrónico derivadas del CETA. A continuación, y con la ayuda de Gowling WLG, firma de abogados asociada a la Cámara, se pasa revista a otros sectores industriales de interés para las empresas europeas, como son aquellos relacionados con la transición ecológica, la industria de alimentación y bebidas, y las tecnologías de digitalización.

\section{Acerca de la EUCCAN}

La EUCCAN lleva veinticinco años apoyando las relaciones comerciales entre la UE y Canadá. Gracias al apoyo de la Delegación de la UE en Canadá, la EUCCAN ha crecido considerablemente en los últimos cuatro años. En la actualidad se está poniendo en marcha una nueva estrategia: ser un firme defensor de las empresas de la UE en Canadá, gracias a la creación de comités industriales para impulsar las oportunidades entre las empresas de la UE y las canadienses.

La EUCCAN comenzó como la organización paraguas de veinticinco cámaras de comercio y asociaciones empresariales estatales de la UE y cuatro miembros asociados que representan a las cámaras de comercio de los países vecinos de la UE. Con el fin de servir y representar mejor los intereses empresariales, la EUCCAN aprobó un nuevo plan estratégico en 2021 y ahora acoge a miembros corporativos de la UE. Como primer paso de esta nueva estrategia, y con motivo de la asamblea general de la organización, Adrian Thomas, presidente nacional de Schneider Electric, ha sido elegido presidente de la EUCCAN.

Apoyada desde 2017 por la Delegación de la Unión Europea en Canadá, la EUCCAN es un recurso clave y un punto de contacto principal para las empresas de la UE en Canadá. La EUCCAN opera en tres pilares clave: defensa, información y representación.

\section{Advocacy}

La EUCCAN defiende a las empresas de la UE en todos los niveles de gobierno (municipal, provincial y federal), identificando los retos y las oportunidades de hacer negocios en $\mathrm{Ca}-$ nadá, abordando asuntos comunes y expresando los intereses empresariales y las recomendaciones a los Gobiernos de la UE y Canadá. Para apoyar estas acciones facilitamos comités industriales, realizamos encuestas y publicamos documentos de posición.

\section{Información}

La EUCCAN informa a las empresas de la UE sobre el CETA proporcionando guías prácticas de negocios, documentos del sector e $\square$ 
información actualizada sobre las relaciones comerciales entre la UE y Canadá. También organizamos seminarios de negocios y eventos de networking en Canadá. Se puede encontrar más información del CETA, en el sitio web de la EUCCAN.

\section{Representación}

La EUCCAN representa los intereses de sus miembros a nivel local e internacional. Ofrecemos a nuestros miembros una mayor visibilidad, facilitamos el acceso y las presentaciones a políticos e instituciones gubernamentales de alto nivel y actuamos como intermediarios ante las principales partes interesadas.

Por supuesto, la EUCCAN sigue dedicada a promover el CETA en la UE y en Canadá para que las empresas se beneficien de sus numerosas posibilidades, como la contratación pública, la movilidad laboral o el comercio electrónico, por mencionar solo estas tres.

\section{Acuerdo Integral Económico y de Comercio entre la Unión Europea y Canadá (CETA)}

Aunque el funcionamiento del CETA se trata en profundidad en otros artículos, es importante mencionar que dicho acuerdo ofrece grandes oportunidades para las empresas de la UE. Pero con demasiada frecuencia las empresas utilizan el CETA por el aspecto comercial, descuidando otras grandes posibilidades que ofrece el acuerdo en sí mismo.

Por lo anterior, este apartado se centra en los siguientes aspectos específicos del CETA:

- Artículo 19: Contratación pública.

- Artículo 16: Comercio electrónico.

\subsection{Contratación pública}

Este inciso se centra en el importante impacto del CETA en la participación de las empresas de la UE en los procesos de contratación pública. Según la Organización para la Cooperación y el Desarrollo Económico (OCDE), los gastos de contratación pública en bienes y servicios del Gobierno de Canadá representan cerca del $33 \%$ de los gastos del Gobierno federal, o algo más del $13 \%$ del PIB de Canadá.

El CETA proporciona a las empresas de la UE acceso no solo a la contratación general de los departamentos y ministerios federales, sino también a la de las provincias y territorios. En el presente artículo solo se tratará el nivel federal.

La sección 19 del CETA, y sus anexos, amplían y garantizan las oportunidades para que las empresas suministren sus bienes, servicios y construcciones a todos los niveles de la Administración y a una amplia variedad de entidades que operan en el sector de los servicios públicos.

A pesar de su amplitud, siguen existiendo ciertas excepciones y barreras. En Canadá, al igual que en la UE, el CETA se aplica únicamente a los contratos de alto valor. Esta norma se impuso para garantizar que las pequeñas y medianas empresas nacionales puedan seguir contratando con el Gobierno para estimular el desarrollo local. Como los umbrales del CETA se expresan en una unidad de reserva del Fondo Monetario Internacional denominada derechos especiales de giro (DEG), los umbrales se ajustan bianualmente para tener en cuenta las fluctuaciones del tipo de cambio entre el dólar canadiense y el DEG.

Global Affairs Canada calcula los umbrales de valor mínimo en dólares canadienses $\triangleright$ 
CUADRO 1

UMBRALES MÍNIMOS DE CONTRATOS EN EL CETA (2020-2021)

(En dólares canadienses)

\begin{tabular}{|c|c|c|c|c|c|}
\hline \multicolumn{2}{|c|}{ Entidades de la Administración central enumeradas } & \multicolumn{3}{|c|}{ Entidades de la Administración central enumeradas } \\
en el anexo 19-3 \\
\hline Productos & Servicios & Construcción & Productos & Servicios & Construcción \\
\hline & & & Sección A & Sección A & \\
238.000 & 238.000 & 9.100 .000 & 650.000 & 650.000 & 9.100 .000 \\
& & Sección B & Sección B & 732.400 \\
\hline
\end{tabular}

para el periodo de tiempo comprendido entre el 1 de enero de 2020 y el 31 de diciembre de 2021, aplicables a la Administración central (Cuadro 1).

Uno de los mayores obstáculos para acceder a las oportunidades del CETA es el desconocimiento de las opciones disponibles y de los métodos utilizados. Para identificar y aprovechar eficazmente las oportunidades que ofrece el CETA, es importante entender las prioridades que generan y el proceso por el que se accede a ellas.

También es importante tener en cuenta que si una empresa de la UE decide operar su negocio en Canadá, a través de una entidad constituida en Canadá, los umbrales mencionados anteriormente no se aplicarán. La constitución de la sociedad otorga a la actividad canadiense de la empresa de la UE el estatus de sociedad canadiense, lo que significa que puede contratar con el Gobierno de Canadá sin las limitaciones del CETA.

La siguiente información se centra en las oportunidades de contratación para las empresas de la UE que no desarrollan su actividad en Canadá a través de una sociedad.

A nivel federal, las oportunidades de contratación se recopilan y se encuentran disponibles en Buyandsell.gc.ca. Este sitio web ofrece información gratuita sobre todas las formas de licitación pasadas y actuales con el Gobierno federal de Canadá. El portal informa si una oportunidad de licitación específica está disponible en el marco del CETA y cuándo están autorizadas las empresas de la UE a presentar propuestas.

Las empresas de la UE deben cumplir básicamente tres requisitos para participar en el proceso de contratación de cualquier agencia o departamento federal.

- La oferta debe ser realizada por una entidad que figure en el CETA.

- La empresa de la UE debe cumplir el límite de valor específico.

- La empresa de la UE solo puede ofertar en mandatos que no entren en ninguna de las exclusiones.

El proceso de adquisición se inicia cuando un departamento o agencia federal envía un pedido a PSPC (Public Services and Procurement Canada). Dependiendo de los requisitos, el pedido puede realizarse a través de un proceso de adquisición competitivo, con el objetivo de obtener el mejor valor para los canadienses con un acceso mejorado que haga hincapié en la competencia y la equidad.

Solo se recurre a un proceso de contratación no competitivo en determinadas circunstancias especiales en las que: 
- La necesidad es una emergencia apremiante en la que el retraso sería perjudicial para el interés público.

- El gasto estimado no supera una determinada cantidad.

- Solo una persona es capaz de realizar la obra, como cuando un proveedor es titular de un derecho de autor o de una licencia.

- La naturaleza del trabajo es tal que no sería de interés público solicitar ofertas (por ejemplo, requisitos relacionados con la seguridad nacional, como algunos proyectos militares).

Para poder contratar con el Gobierno federal, las empresas de la UE deben registrarse en el Sistema de Información de Registro de Proveedores (SRI) y obtener un número de negocio de adquisición (PBN) de 15 dígitos. Antes de poder registrarse en el SRI, una empresa no residente debe obtener un número de empresa $(B N)$ emitido por la Agencia Tributaria Canadiense (CRA). El registro en el SRI hace que el nombre de la empresa y su capacidad de suministro estén ampliamente disponibles para los departamentos y organismos federales, que pueden utilizar el SRI para identificar las fuentes de suministro de los bienes y servicios que necesitan.

Es esencial que una empresa de la UE promueva de forma proactiva su negocio como proveedor, destacando los bienes y servicios que proporciona y haciendo hincapié en las razones por las que el Gobierno debería elegir a su empresa como proveedor. Cuando una empresa está preparando una propuesta para una licitación, es esencial asegurarse de que los departamentos y organismos conozcan las capacidades especiales y las características diferenciadoras que pueda tener esa empresa, que la distingue de sus competidores.
El Gobierno canadiense busca productos o servicios respetuosos con el medioambiente y anima a sus organismos y departamentos a comprar bienes y servicios ecológicos que ayuden o, al menos, no le perjudiquen. Esta información debe mencionarse en una propuesta a la posible autoridad contratante.

Los departamentos y organismos federales están obligados a utilizar Buyandsell.gc.ca para anunciar sus requisitos para las oportunidades de licitación sujetas a cualquiera de los acuerdos comerciales de Canadá.

En Buyandsell.gc.ca los proveedores pueden buscar oportunidades pasadas y nuevas, y también proveedores precalificados. Utilizar el código GSIN de bienes y servicios correspondiente es otra forma de filtrar las oportunidades de interés y de registrarse para recibir notificaciones una vez que las oportunidades bajo una categoría específica estén disponibles.

El Gobierno federal puede contratar de muchas maneras. La PSPC utiliza varios métodos para solicitar ofertas, como RFQ, ITT y RFP, entre otros. Cada método de contratación tiene sus propios requisitos y los proveedores deben consultar Buyandsell.gc.ca para estar al tanto de los requisitos que PSPC anuncia públicamente en relación con una adquisición de un bien, servicio o construcción específicos.

Cada licitación es única. Por ello se anima a las empresas de la UE a leer el pliego de condiciones con mucha atención y responder a lo que se les pregunta en las diferentes secciones:

- Sección técnica. Aquí las empresas tienen la oportunidad de describir qué y cómo van a realizar el contrato si se les adjudica. La sección técnica suele comenzar con una breve introducción en la que se evalúa la situación actual y la necesidad del proyecto, los objetivos del $\triangleright$ 
trabajo propuesto, las razones para llevarlo a cabo como se propone y los beneficios que se derivarán de él. Se indica el plan de trabajo, la metodología, las técnicas, la viabilidad y el grado de éxito esperado, y se identifican los problemas previstos y el plan de contingencia en caso de que surjan, las tareas y los resultados específicos y el calendario de finalización o entrega.

- Sección de gestión. Aquí es donde se presenta al equipo y se explica cómo cumplen las cualificaciones requeridas, normalmente mediante currículos. Si el trabajo que se va a realizar es en régimen de subcontratación, aunque sea parcial, debe facilitarse la misma información sobre cada subcontratista.

- Sección financiera. Esta sección está diseñada para proporcionar un desglose detallado del precio cotizado y cualquier elemento de coste. El pliego de condiciones explicará los elementos de coste que se tendrán en cuenta en la evaluación financiera.

Si hay alguna pregunta sobre la oportunidad que se ofrece, puede presentarse por escrito. Las modificaciones que se realicen en el pliego de condiciones estarán disponibles en Buyandsell.gc.ca.

A la hora de preparar una propuesta de licitación, las empresas deben ser conscientes de ciertos requisitos adicionales exigidos desde el Gobierno. Una prueba de ello son las autorizaciones de seguridad o security clearances. Estas autorizaciones pueden ser necesarias cuando un contrato de compra requiere que una empresa acceda a información clasificada, a activos o a lugares de trabajo federales de carácter protegido.
De manera habitual, el pliego de condiciones especificará el nivel de seguridad requerido; además, es importante mencionar que la obtención de una autorización de seguridad suele ser un proceso largo y tedioso, por lo que se anima a las empresas a hablar con el funcionario de contratación lo antes posible para iniciar el proceso.

Asimismo, las empresas deben familiarizarse con las Cláusulas y Condiciones Estándar de Adquisición (Standard Acquisition Clauses and Conditions, SACC por sus siglas en inglés) y revisar cuidadosamente los términos, condiciones e instrucciones estándar que normalmente se incluyen en cada documento de solicitud en texto completo o por referencia.

Llegado el momento de la adjudicación, solo se considerarán las ofertas que cumplan los criterios técnicos obligatorios; en este sentido, el precio será el factor determinante de una selección cuando el pliego así lo especifique, sin embargo, la elección también podría sustentarse en otros criterios, como la oferta de mayor valor o la oferta técnica más alta dentro de un presupuesto estipulado.

\subsection{Comercio electrónico}

Por medio de este apartado se pretende ofrecer una visión general, no exhaustiva, acerca de las leyes y reglamentos aplicables al comercio electrónico en Canadá. Su objetivo es destacar algunas de las cuestiones fundamentales que las empresas europeas deben conocer de cara a futuras oportunidades de negocio en las que el comercio electrónico se encuentre involucrado.

En este sentido, el artículo 16.3 hace referencia a una prohibición sobre los derechos aduaneros, por medio de la cual se establece que los Estados firmantes no impondrán $\triangleright$ 
derechos, tasas o recargos a las «entregas electrónicas», incluidos los programas informáticos, los textos, los vídeos, las imágenes, las grabaciones de sonido y otros suministros de carácter digital.

Por su parte, el artículo 16.4 trata sobre la privacidad de los datos de los usuarios del comercio electrónico y exige que Canadá y la UE tengan en cuenta las normas internacionales en materia de protección de datos. En concreto, pide a los Estados firmantes que reconozcan la importancia de la interoperabilidad de los marcos normativos entre las distintas naciones para facilitar el uso del comercio electrónico, especialmente para las pequeñas y medianas empresas.

En Canadá, la mayoría de las leyes aplicables al comercio electrónico son de carácter provincial; entre ellas se encuentran las leyes relativas a la protección de los consumidores y las que rigen los contratos. Como cada provincia tiene jurisdicción sobre estas áreas del derecho, es de vital importancia que las empresas de comercio electrónico estén familiarizadas con las leyes de cada provincia en la que hacen negocios.

Por su parte, las leyes federales también se aplican en ciertos aspectos críticos del comercio electrónico; ejemplo de esto son las leyes que regulan la propiedad intelectual, la competencia y el envío de comunicaciones comerciales. Algunos aspectos del funcionamiento de una empresa también pueden estar regidos por leyes federales y provinciales, como la privacidad y la seguridad de los datos.

En Canadá, la venta de productos y/o servicios a través de canales de comercio electrónico plantea una importante cuestión sobre el ámbito de competencia de los tribunales locales en aquellos litigios derivados de una transacción realizada a través de internet. Dicho esto, para evitar que existan extralimitaciones, los tribunales canadienses han establecido varias pruebas para determinar si existe una conexión real entre Canadá y el demandado o el objeto del litigio. La siguiente lista enuncia los factores a considerar:

- El lugar de residencia del demandado.

- El lugar en el que el demandado ejerce su actividad.

- El lugar de celebración del contrato relacionado con el litigio.

En este sentido, en el comercio tradicional, la aplicación de esta prueba está detallada en la jurisprudencia canadiense mientras que en el contexto del comercio electrónico pueden surgir problemas específicos, como, por ejemplo, las comunicaciones electrónicas que cruzan múltiples fronteras nacionales y se producen en más de un país.

Entre los factores relevantes también se incluiría la ubicación del proveedor de contenidos, el servidor anfitrión, los intermediarios y el usuario final, además de los criterios tradicionales de la prueba de «conexión real y sustancial». Por lo general, un tribunal canadiense será competente cuando el foro sea el lugar de transmisión o el lugar de recepción. Por lo tanto, habitualmente, las empresas extranjeras que realizan comercio electrónico en Canadá serán juzgadas por los tribunales canadienses por el hecho de estar situadas en un país extranjero.

Además, está bien establecido que cuando la aceptación de un contrato se transmite de forma electrónica e instantánea, este se considera realizado en la jurisdicción en la que se recibe la aceptación. Por lo tanto, para las empresas de comercio electrónico que celebran contratos con clientes $u$ otras partes $D$ 
situadas en Canadá, todos esos contratos tendrían probablemente una "conexión real y sustancial» en virtud de la conexión entre el contrato y la provincia en la que se realizó.

Por último, y a modo de conclusión, al poner a disposición de las empresas y consumidores canadienses productos y servicios por medio del comercio electrónico, las empresas extranjeras deben ser conscientes de que la mayoría de las leyes existentes que se aplican al comercio tradicional también se aplicarán a sus operaciones. Esto incluye las leyes que rigen la propiedad intelectual, la privacidad, la protección del consumidor, la fiscalidad, la publicidad, la importación o exportación, la seguridad de los productos y sus normas.

El cumplimiento de las leyes de privacidad sigue siendo uno de los mayores retos a los que se enfrenta el comercio electrónico en la actualidad. Los individuos y las organizaciones son cada vez más sensibles a la hora de proporcionar información y datos personales a terceros. Las leyes que rigen la recopilación, el uso y la divulgación de la información personal imponen restricciones aún mayores sobre la forma en que las organizaciones pueden utilizar esta información. Por su parte, las empresas de comercio electrónico que realizan operaciones transfronterizas también deben ser conscientes de las leyes de cada jurisdicción, ya que los requisitos de cada una de ellas pueden ser diferentes.

Las empresas de comercio electrónico, en sus funciones comerciales, recaban grandes volúmenes de información personal, como el nombre y la dirección de una persona cuando interactúa con el sitio web de la empresa o mediante técnicas de recopilación de datos, como las cookies, que rastrean a los usuarios mientras navegan por un sitio web. Estas mismas empresas pueden utilizar la información personal y demográfica de los usuarios para crear perfiles personales detallados de las preferencias de estos, y así dirigir sus esfuerzos publicitarios de forma personalizada. A causa de lo anterior, el marco legal canadiense en materia de privacidad restringe el modo en que estas empresas pueden recoger, utilizar o revelar información personal.

Para ahondar en el tema, en Canadá, la protección de la información personal se rige por dos leyes federales. La Ley de Privacidad (The Privacy Act), que se ocupa del uso de la información personal por parte de las entidades del Gobierno federal, y la Ley de Protección de la Información Personal y los Documentos Electrónicos (The Personal Information Protection and Electronic Documents Act, o PIPEDA), que se aplica a las entidades del sector privado, incluidas las organizaciones extranjeras que hacen negocios en Canadá. En este sentido, algunas provincias han promulgado leyes de privacidad consideradas "sustancialmente similares» a la PIPEDA, como es el caso de Quebec y Alberta.

Las empresas que realizan comercio electrónico en Canadá deben respetar las estrictas normas de la PIPEDA sobre la forma en que todas las entidades del sector privado pueden recopilar, utilizar o revelar información personal en el curso de sus actividades comerciales.

La información personal se define como «información sobre un individuo identificable, pero no incluye el nombre, el cargo o la dirección comercial o el número de teléfono de un empleado de una organización». Al igual que el Reglamento General de Protección de Datos (RGPD) de la UE, la PIPEDA impone restricciones sobre la forma en la que una organización puede recopilar, utilizar y divulgar información personal. En general, la PIPEDA exige que las $\triangleright$ 
organizaciones notifiquen y reciban el consentimiento de las personas de las que recogen datos personales.

Asimismo, la PIPEDA también exige que las organizaciones sean transparentes en cuanto a sus prácticas de tratamiento de la información personal. Aunque no prohíbe a las organizaciones transferir información personal a otras jurisdicciones para su tratamiento, la organización que la transfiere seguirá siendo responsable de la información enviada al extranjero y deberá cumplir la legislación canadiense sobre privacidad.

Al igual que en el caso del RGPD, la PIPEDA exige a las organizaciones que informen directamente a la Oficina del Comisionado de Privacidad de Canadá (Office of the Privacy Commissioner of Canada) en caso de que se produzca una brecha de seguridad que ponga en compromiso la información personal de las personas afectadas. De igual manera, las organizaciones también deben notificar a las personas afectadas y, en determinadas circunstancias, a los terceros pertinentes. Además, deben informar a dicha oficina, tan pronto como sea posible, cuando una organización haya detectado que se ha producido una violación de datos.

Por último, las organizaciones están obligadas a notificar a cualquier otra organización o institución gubernamental si creen que dicho organismo puede reducir el riesgo o mitigar el daño. Las organizaciones deben mantener un registro de todas las violaciones de la información personal y proporcionar una copia de los registros a la Oficina del Comisionado de Privacidad a petición. No informar a sabiendas a la Oficina del Comisionado de Privacidad o no notificar a las personas afectadas puede dar lugar a multas de hasta 100.000 dólares canadienses.
Las firmas electrónicas se han hecho cada vez más populares para la formalización de documentos comerciales, ya que eliminan la necesidad de asistir a reuniones en persona o de enviar por correo los documentos a firmar. De este modo, las firmas electrónicas pueden acelerar las transacciones comerciales y proporcionar una mayor comodidad a las partes que realizan la transacción.

Debido a su creciente popularidad, todas las provincias y territorios canadienses cuentan actualmente con una ley de comercio electrónico que, en general, se ajusta a la Ley Modelo de las Naciones Unidas sobre Comercio Electrónico.

Con la excepción de un número limitado de circunstancias que se describen a continuación, las transacciones y los contratos celebrados por vía electrónica en Canadá son tan legalmente exigibles como sus equivalentes en papel. Cada ley provincial de comercio electrónico establece que los acuerdos firmados electrónicamente deben cumplir los requisitos convencionales del derecho contractual, como la oferta, la aceptación y la contraprestación. Las circunstancias en las que no se puede confiar en las firmas electrónicas incluyen los testamentos, los poderes, los instrumentos negociables y los fideicomisos creados por testamentos.

También hay soluciones de software de terceros para los comerciales que deseen ejecutar documentos por vía electrónica. Siempre que las firmas electrónicas sean claramente identificables y las partes hayan consentido el uso de firmas electrónicas, el uso de software de terceros será generalmente aceptado en los acuerdos comerciales. Sin embargo, las organizaciones deben ser conscientes de ciertos casos en los que las firmas electrónicas no son suficientes. Esto puede incluir documentos $\triangleright$ 
de financiación para los que algunas instituciones financieras o partes prestamistas pueden exigir firmas de tinta húmeda en función de sus políticas internas.

Aunque el dominio .com es el más común del mundo, muchas empresas canadienses optan por utilizar un dominio .ca para fomentar los vínculos nacionales y la confianza con los clientes locales.

La elección de un dominio .ca también puede mejorar los resultados de búsqueda locales. Sin embargo, un dominio .com puede tener mejores resultados a nivel internacional debido a su naturaleza genérica y universal.

El dominio .ca está gestionado por la Autoridad Canadiense de Registro de Internet (CIRA).

Sin embargo, la mayoría de los nombres de dominio se compran a registradores o revendedores. La CIRA describe los siguientes pasos para registrar un dominio .ca:

- Buscar el nombre de dominio .ca deseado a través del sitio web de CIRA.

- Elegir un registrador o revendedor.

- Registrar y asegurar el dominio .ca

Para registrar un nombre de dominio .ca, la empresa interesada debe cumplir los Canadian Presence Requirements, que garantizan que el espacio del dominio .ca se constituye para el desarrollo social y económico de todos los canadienses. En virtud de estos requisitos, solo determinadas personas y entidades podrán solicitar, poseer y mantener un nombre de dominio .ca.

En el contexto del comercio electrónico, algunas opciones aplicables pueden ser las siguientes:

- Ser ciudadano canadiense o residente permanente en Canadá.
- Ser una corporación constituida bajo las leyes de Canadá o de cualquier provincia o territorio de Canadá.

- Ser una sociedad registrada bajo las leyes de cualquier provincia o territorio de Canadá.

- Ser una organización no constituida en sociedad, una asociación o un club en el que al menos el $80 \%$ de sus miembros residan habitualmente en Canadá y el $80 \%$ de sus directores y otros representantes residan de forma habitual en Canadá.

- Ser un propietario de una marca, pero solo en la medida en que el nombre de dominio .ca consista en la redacción exacta de la marca registrada.

La CIRA y sus registradores certificados tienen la responsabilidad de solo comunicar las actualizaciones y avisos de renovación a los solicitantes de registro y a sus contactos administrativos, por lo que se recomienda a las empresas que registren su dominio .ca por sí mismas y no a través de un tercero, como un agente o un desarrollador de sitios web.

En Canadá, cada provincia y territorio tiene su propia legislación de protección al consumidor, que es convenientemente aplicada a las transacciones que involucran a los consumidores ubicados en esa jurisdicción. Por ello, una consideración clave para las organizaciones que llevan a cabo alguna forma de comercio electrónico en Canadá es garantizar el cumplimiento de la legislación de protección del consumidor de la provincia o territorio correspondiente.

De la misma manera, cada provincia canadiense tiene requisitos específicos que deben cumplirse cuando se realizan transacciones online. Estos requerimientos, que difieren tanto de una provincia a otra como del producto $\circ \square$ 
servicio sobre el que versa el acuerdo, incluyen igualmente la exigencia de que la compañía proporcione al usuario información específica sobre la transacción, así como la necesidad de que la organización siga los procedimientos específicos del canal electrónico.

Las empresas que utilizan el comercio electrónico para poner a disposición sus productos y servicios confían en el correo electrónico para comunicarse con los clientes potenciales y existentes. La comunicación por este tipo de medio ofrece a las empresas tanto una solución de bajo coste para llegar a un número importante de personas como la posibilidad de promocionar productos nuevos o existentes. Sin embargo, es esencial comprender las leyes que se aplican a los mensajes electrónicos comerciales, pues, en caso de no ser respetados, las compañías se arriesgan a recibir importantes multas y a sufrir daños en su reputación.

La legislación antispam de Canadá (CASL) es una de las más prescriptivas del mundo. La CASL regula las comunicaciones electrónicas comerciales no solicitadas (CEM) enviadas por entidades a particulares, así como la instalación no solicitada de software, y su incumplimiento puede dar lugar a importantes sanciones monetarias y cargos penales. Por ello, los particulares y las organizaciones deben asegurarse de que no envían mensajes electrónicos comerciales que quebranten sus preceptos.

La CASL se aplica tanto a las comunicaciones electrónicas a las que se accede en Canadá como a aquellas que se envían desde un dispositivo situado en el país. De la misma manera, y dado que las empresas también pueden ser responsables de los CEM que proveedores terceros envíen en su nombre, son estas las que deben asegurarse de que sus proveedores de servicios la cumplan de manera íntegra.
En concreto, prohíbe el envío de CEM sin consentimiento previo, y lo limita solamente a aquellos que cumplen determinados requisitos formales. De igual forma, esta legislación impide la instalación de software en los ordenadores de destinatarios o propietarios sin su consentimiento previo, hecho que puede ser proporcionado expresamente por el individuo o, en determinadas circunstancias, de forma implícita. En lo referente a este último aspecto, la CASL es prescriptiva en cuanto a la forma en que un destinatario puede dar su consentimiento expresamente, exigiendo el opt-in del destinatario antes de recibir cualquier CEM, y exige que la información de contacto de la organización que solicita el consentimiento sea proporcionada al destinatario en el momento en que lo hace.

La CASL define los CEM como mensajes electrónicos que fomentan la participación en «actividades comerciales», independientemente de cualquier expectativa de beneficio. Esta definición es extremadamente amplia y puede incluir los correos electrónicos promocionales o los que anuncian descuentos. Por ello contiene una lista limitada de circunstancias específicas y plazos estrictos en los que un remitente podría basarse en el consentimiento implícito para enviar CEM.

No obstante, resulta aconsejable que las compañías no traten de basar sus comunicaciones en el consentimiento implícito, debido fundamentalmente a la limitación temporal que esta tiene. Del mismo modo, aquellas que lo hagan deben mantener registros y hacer un seguimiento de cuándo el consentimiento implícito deja de ser válido, así como asegurarse de que se deja de enviar CEM a esa persona inmediatamente después de que el consentimiento implícito en el que se apoya haya expirado. 
Además, la CASL exige que todo envío de CEM incluya un mecanismo de cancelación de la suscripción para que el destinatario pueda optar por no recibir más en el futuro, así como la inclusión de cierta información prescrita sobre el remitente. Este mecanismo debe permitir al destinatario darse de baja fácilmente y sin coste alguno de futuras comunicaciones electrónicas y dar al remitente hasta diez días a partir de la solicitud de cancelación de la suscripción para dejar de enviar este tipo de mensajes.

\section{La transición ecológica de Canadá}

Bajo el compromiso de llevar a cabo una transición a una economía de bajas emisiones, los Gobiernos canadienses están trabajando con empresas y partes interesadas de todo el mundo para implementar e incorporar tecnologías limpias al mercado. Según Invest in Canada, el país es el lugar óptimo para este tipo de desarrollos debido a sus «bajos costes [de investigación y desarrollo], su mano de obra altamente cualificada, su riqueza en recursos naturales y su apoyo a la innovación».

Canadá es un polo de transformación de tecnología verde en los sectores del transporte, las infraestructuras y la construcción. Así, el país se está convirtiendo en una economía líder en el desarrollo de energías renovables, tecnologías de captura de carbono, electrificación de vehículos y fuentes de combustible innovadoras. Programas como el Fondo de Innovación Estratégica, dotado con 8.000 millones de dólares, Acelerador Net Zero o el Plan de Crecimiento del Banco de Infraestructuras de Canadá (CIB), que asciende a 10.000 millones de dólares, están apoyando proyectos que pretenden descarbonizar la industria pesada, apoyar las tecnologías limpias y ayudar a acelerar significativamente la reducción de las emisiones nacionales de gases de efecto invernadero para 2030.

Recientemente, el Gobierno de Canadá ha anunciado una serie de inversiones y normativas para ayudar a las empresas e industrias a realizar la transición hacia la venta de vehículos con cero emisiones para 2035. Ejemplo de ello es la inversión de más de mil millones de dólares para apoyar el desarrollo de cargadores rápidos para vehículos eléctricos, la puesta en marcha de estaciones de gas natural a lo largo de los principales corredores de mercancías y de estaciones de hidrógeno en centros metropolitanos de todo el país. Igualmente, se han introducido nuevas medidas para apoyar a los fabricantes de automóviles, incluida una rebaja del impuesto de sociedades del $50 \%$.

También han llegado importantes inversiones a los sectores de las infraestructuras y de la construcción con el propósito de alcanzar los objetivos de emisiones netas cero en 2050 . Estos fondos apoyan la generación de energía sostenible, la reconversión de edificios energéticamente eficientes y la infraestructura de banda ancha sostenible. Además, como informa el Instituto Internacional para el Desarrollo Sostenible, una parte de esta inversión se destina a acelerar la planificación y ejecución de infraestructuras, por ejemplo, proporcionando importantes estudios de viabilidad y orientación técnica.

Canadá se ha comprometido a aprovechar los beneficios de la eficiencia energética en todos los sectores. Además de la financiación específica para proyectos de eficiencia, se ha unido recientemente al «Club del $3 \%$ », un colectivo de países, empresas e instituciones que se han comprometido a lograr un aumento del $\triangleright$ 
tres por ciento en la eficiencia energética cada año. Estos aumentos son realmente significativos, dado que la Agencia Internacional de la Energía determina que las políticas de eficiencia podrían aportar más del $40 \%$ de las reducciones de emisiones necesarias para alcanzar los objetivos del Acuerdo de París.

En tanto que la comprensión de los requisitos legales y reglamentarios por parte de los inversores y las empresas suponga que sea más fácil aprovechar las oportunidades de negocio, es clave conocer los matices y las complejidades del panorama normativo canadiense. Así, en cuanto al tema sobre el que versa este epígrafe, es clave tener en cuenta la multiplicidad de Gobiernos -federal, provincial, territorial y municipal— que promulgan y regulan las leyes medioambientales a lo largo del país.

La legislación medioambiental abarca una serie de ámbitos, tales como el cambio climático, la gestión de emisiones, la energía, la gestión de residuos, el tratamiento del agua, las especies en peligro, los residuos, los terrenos contaminados, las sustancias tóxicas, la pesca, la responsabilidad social de las empresas, la gestión de riesgos y la planificación y la evaluación del impacto medioambiental. Una falta de consideración o de gestión escrupulosa del cumplimiento de estas leyes podría ocasionar una pérdida de oportunidades y la generación de daños financieros y de reputación para las empresas. Por todo ello, los riesgos ambientales, sociales y de gobernanza (ASG) se están convirtiendo, cada vez más, en un foco de atención para los accionistas. Además, las decisiones de inversión están basadas en datos y en una planificación que tiene en cuenta las evaluaciones de riesgo y las estrategias a largo plazo.

\section{Industria alimentaria y de bebidas}

La industria alimentaria y de bebidas de Canadá es una de las más sólidas del mundo. Gracias a un sistema normativo diligente y tremendamente eficaz que supervisa cada paso de la cadena de suministro de alimentos y bebidas —desde la fabricación y la exportación hasta la importación y la distribución-, Canadá es considerado continuamente como un país valioso, fiable y acogedor con el que hacer negocios en el ámbito de los alimentos y las bebidas.

En el plano institucional, el Ministerio de Salud de Canadá es la principal entidad gubernamental responsable de regular la industria de alimentos y bebidas del país, incluyendo la creación de normas específicas que garantizan que este tipo de productos son seguros para el consumo y nutritivos. Asimismo, la Agencia Canadiense de Inspección Alimentaria es el organismo gubernamental responsable de supervisar las normas de salud y seguridad de los alimentos y bebidas que se venden en Canadá, incluida la regulación de todos los envases, el etiquetado y la publicidad de alimentos y bebidas.

Por otra parte, una de las características que convierten al panorama normativo canadiense en excepcionalmente único es su enfoque global a la hora de crear y aplicar las normas y reglamentos que afectan a la industria de la alimentación y las bebidas. Así, la alineación con el enfoque de los principales socios comerciales de Canadá, incluida la Unión Europea, es de suma importancia para las entidades responsables de mantener y hacer cumplir estas medidas reglamentarias. Por ello, se determina que, solo a través de la comprensión y apreciación de estos grandes socios comerciales, Canadá puede garantizar el éxito en su propio ámbito. 
En la última década, Canadá ha ido dando importantes pasos en la modernización de sus normas y reglamentos específicos para la industria de alimentos y bebidas. Ejemplo de ello es el reciente cambio que el país se ha propuesto implementar para antes de finales del año 2022 y que traerá consigo una profunda transformación en el etiquetado de estos productos, haciendo que los datos nutricionales y las declaraciones de ingredientes sean más racionales y comprensibles, así como exigiendo la especificación de colores añadidos a los distintos productos.

De igual manera, y con el objetivo de ampliar la gama de alimentos de origen vegetal disponibles en el mercado canadiense, el país también está a punto de establecer políticas modernizadoras con respecto a este tipo de productos. Así, a medida que la población se replantee su consumo de productos animales, se espera que la demanda de alimentos de origen vegetal en Canadá crezca exponencialmente en los próximos años.

Recientemente, el Ministerio de Sanidad de Canadá ha publicado una propuesta de normativa para los alimentos complementados. Los alimentos complementados son aquellos productos alimenticios y bebidas que contienen uno o más ingredientes complementarios añadidos, como vitaminas, minerales y cafeína. Aunque estas propuestas de reglamentación se encuentran todavía en su fase inicial, es muy importante que las empresas de alimentos y bebidas de todo el mundo que deseen introducirse en el mercado canadiense en los próximos años estén atentas a estas novedades y a cómo pueden afectar a sus productos en el futuro. En la fecha de realización de este artículo, la normativa sobre alimentos complementados estaba todavía en la fase inicial de consulta.
Con una población diversa y progresista de aproximadamente 38 millones de personas y un hambre insaciable de más opciones alimentarias, Canadá representa una oportunidad excepcional para que las empresas de alimentación y bebidas amplíen su alcance. Con la aplicación de normativas modernizadas y nuevos e interesantes protocolos en el horizonte, nunca ha habido un mejor momento para entrar en este vasto mercado.

\section{Enfoque digital}

Canadá es un líder mundial en materia de tecnología. Su compromiso con la innovación, los incentivos gubernamentales para potenciar la investigación y el desarrollo (I+D), la calidad de su cantera de talentos tecnológicos, su creciente número de centros tecnológicos de categoría mundial y su acceso al mercado estadounidense convierten a Canadá en uno de los principales lugares del mundo para hacer negocios para las empresas tecnológicas y los inversores.

Con la reputación de tener mano de obra tecnológica altamente cualificada, las empresas tecnológicas globales están apuntando a ciudades canadienses como Montreal, Vancouver y el Corredor Tecnológico de Innovación entre Toronto y Waterloo para su expansión y crecimiento.

Canadá ha redoblado sus esfuerzos para invertir en innovación y desarrollo en los últimos años, creando y financiando una iniciativa de superclústeres en todo el país para impulsar la innovación y crear un modelo de colaboración para la I+D. Los superclústeres de tecnología digital y de IA a escala, por nombrar algunos, han dado lugar a la creación de tecnologías y productos que sitúan a Canadá en el mapa mundial de la innovación. 
De igual manera, el país sigue atrayendo a las empresas tecnológicas e invirtiendo en ellas por sus competitivos costes empresariales, sus razonables tipos impositivos y sus programas de incentivos respaldados por el Gobierno. El incentivo fiscal para la investigación científica y el desarrollo experimental (SR\&ED) es un ejemplo de los programas de incentivos disponibles para las empresas canadienses que realizan actividades de $\mathrm{I}+\mathrm{D}$, incluyendo la creación y/o mejora de tecnologías nuevas y existentes.

Aunque la COVID-19 aceleró la digitalización a nivel mundial, Canadá sigue estando a la cabeza de los avances tecnológicos con un fuerte enfoque en la inteligencia artificial (IA), blockchain, comercio electrónico, fintech, ciberseguridad y privacidad. En concreto, Canadá ha experimentado un crecimiento significativo en la adopción de tecnologías fintech, así como un crecimiento exponencial de la inversión extranjera en ese campo, lo que ha llevado a un aumento de los productos con ellas relacionadas en el mercado nacional.

El Gobierno canadiense también ha dado un paso importante para fomentar la innovación y avanzar en la transformación digital en Canadá con su anuncio del proyecto de ley C-11 para modernizar la legislación relacionada con la privacidad de los datos. La legislación propuesta sustituirá las actuales leyes de privacidad del sector privado por un nuevo marco legal de privacidad y datos, cuyo objetivo es lograr un equilibrio entre la protección de la información personal de los consumidores y permitir que la industria y la innovación sigan prosperando. Esta nueva legislación, en caso de entrar en vigor, añadirá protecciones adicionales relacionadas con el manejo de la información digital, requisitos de consentimiento mejorados, múltiples excepciones a los requisitos de consentimiento para permitir la flexibilidad, así como aclarar las responsabilidades de los proveedores de servicios e introducir la equidad procesal mediante el establecimiento de un tribunal al que las empresas puedan recurrir las decisiones. Aunque el proyecto de ley C-11 aún está en sus primeras fases, resulta evidente que se avecinan cambios positivos en el ámbito de la privacidad en Canadá.

Con un ecosistema tecnológico sin parangón, programas e incentivos gubernamentales innovadores y una mano de obra tecnológica de excepcional talento, Canadá es el lugar ideal para que las empresas y los inversores entren en el mercado tecnológico.

\section{Bibliografía}

European Union Chamber of Commerce in Canada. (2020). Government procurement in Canada. CETA: opportunities and barriers for European Union companies. https://euccan.com/wp-content/uploads/ 2020/06/Governement-Procurement-in-Canada-EUCCAN-June-2020.pdf

European Union Chamber of Commerce in Canada. (2021). CETA \& Ecommerce: considerations for EU companies operating in Canada. https://euccan.com/wp-content/uploads/2021/02/CETA-E-Commerce_2021-LD.pdf 
